\title{
Simulation aided design of a low cost ion mobility spectrometer based on printed circuit boards
}

\begin{abstract}
Miniaturized low-cost drift tubes with high analytical performance are a key component for the design of powerful and mass-deployable hand-held ion mobility spectrometers. Thus, a simple model that estimates the influence of the geometrical dimensions on the analytical performance is highly desirable for an effective design process. In this work, we present a simple procedure to predict peak distortion based on only the electrical field distribution inside the drift tube, which can be rapidly simulated using the finite element method. A simulation of the ion motion is not required. Based on these results, we developed an ion mobility spectrometer manufactured entirely from standard printed circuit boards (PCB). Since no additional components were used apart from electrical and gas connectors, ion source and metal grids, the presented ion mobility spectrometer is very simple and inexpensive. Nevertheless, the design provides a resolving power of 82 at a drift length of $50 \mathrm{~mm}$ and a drift voltage of $3 \mathrm{kV}$ using a tritium ion source and a field switching shutter. The limits of detection for one second of averaging are $80 \mathrm{ppt}_{\mathrm{v}}$ for acetone, $35 \mathrm{ppt}_{\mathrm{v}}$ for dimethyl methylphosphonate and $180 \mathrm{ppt}_{\mathrm{v}}$ for methyl salicylate.
\end{abstract}

\section{Introduction}

An ion mobility spectrometer (IMS) is a device for detecting trace gases in air originally developed for detection of explosives and drugs [1]. Within the last decade, IMS entered new markets, for example in medical applications [2; 3] or as detectors for chemicals in industrial environments [4]. To reach an even wider field of possible applications, many research groups are working on new techniques to improve the performance of IMS. However, to push IMS in the mass-deployable hand-held sector, the main focus are basically low prices and a compact and robust design. Therefore, we developed a design which is suitable for low cost and portable applications while maintaining the resolving power of laboratorygrade systems.

The ion separation in a drift tube IMS is the result of ion motion along the axis of the drift tube driven by a homogeneous electrical field. To initiate the measurement, a packet of ions is injected into the drift tube. During their motion, the ions are separated based on their mobility in the present drift gas. At the end of the drift tube, the ions are captured by a detector which converts and amplifies the ion current into a measurable voltage. By plotting the measured voltage over the drift time, i.e. the time the ions need to reach the detector, a spectrum is obtained. Without interferences, each ion species will create a Gaussian shaped peak in the spectrum, which can be used to identify the components of the ionized gas.

Two important parameters which characterize the performance of an IMS are the resolving power $\left(R_{p}\right)$ and the limits of detection (LOD). The resolving power is defined as the quotient of the drift time $t_{d}$ and the full width at half maximum ( $w_{F W H M}$ ) of the corresponding Gaussian peak in the spectrum.

$$
R_{p}=\frac{t_{d}}{w_{F W H M}}
$$


Therefore, it is a measure for the ability to separate substances with similar mobilities. A low resolving power might lead to a spectrum with overlapping peaks, while a high resolving power yields a clear separation of all visible components. The limit of detection refers to the systems capability to detect a given substance. Typically, the LOD is defined as the concentration generating a signal amplitude equal to three times the standard deviation of the noise. Thus, a large signal-to-noise-ratio (SNR) is essential for achieving good limits of detection.

\section{Simulation based design process}

A typical drift tube IMS basically consists of three parts - the ion source, the drift tube and the detector. Of these components, the drift tube typically is the largest, includes the highest number of components and therefore most likely provides the highest optimization potential.

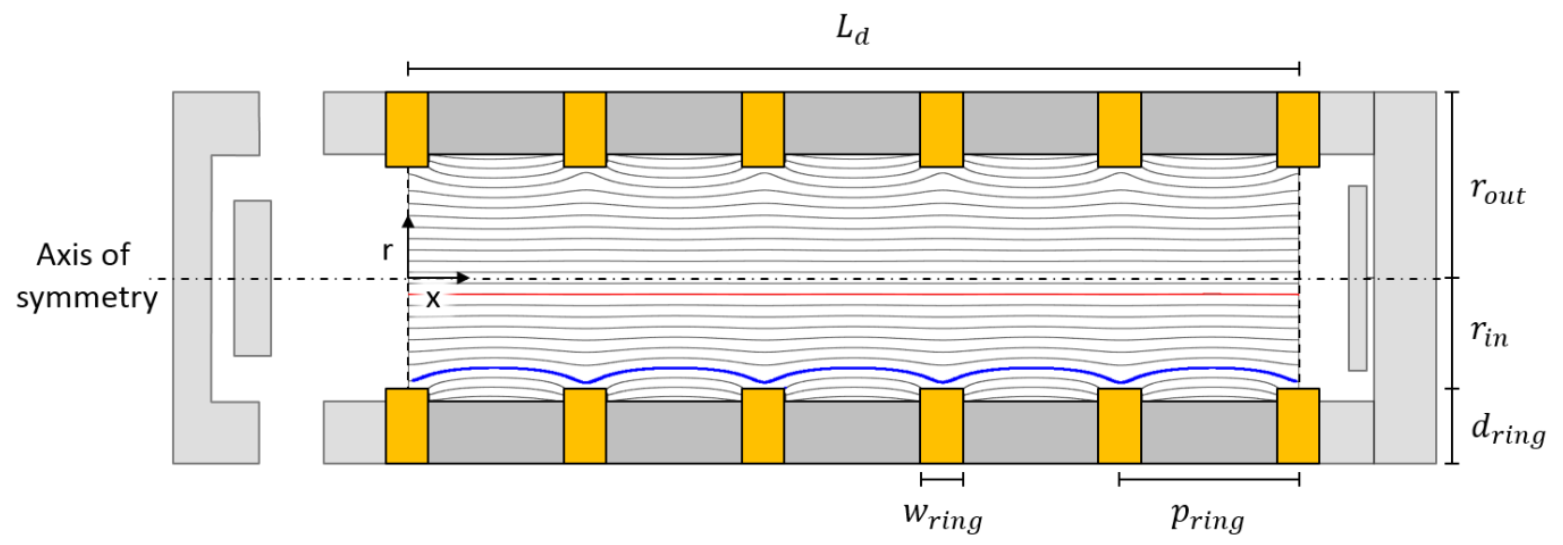

Figure 1: Distribution of the electrical field $E_{(x, r)}$ in a simplified geometrical model. The length of the drift tube $L_{d}$ is determined by the number of rings $n_{\text {ring }}$ and the pitch $p_{\text {ring }}$ between two guard ring centers. The outer radius of the tube $r_{\text {out }}$ is given by the ring depth $d_{\text {ring }}$ and the inner radius $r_{i n}$.

The required space for the drift tube is determined by its length $L_{d}$ and its outer radius $r_{\text {out }}$. Since the systems resolving power is determined by the length $L_{d}$ and the peak width due to injection $w_{\text {inj }}$ and amplifier $w_{a m p}$ as shown in eq. ( 2 ), we cannot reduce the length without improving the ion shutter and amplifier if a constant resolving power is required [5].

$$
R_{p} \sim \sqrt[3]{\frac{L_{d}^{2}}{\sqrt{w_{i n j}^{2}+w_{a m p}^{2}}}}
$$

However, there is no immediate impact of the outer radius $r_{\text {out }}$ on the resolving power. Nevertheless, a homogenous electrical field is imperative for maintaining IMS resolving power, thus creating an indirect influence of the outer radius on the resolving power. Therefore, it is necessary to analyze the relationships between $r_{\text {out }}$ and the electrical field distribution $E_{(x, r)}$, which is illustrated inside the drift tube in Figure 1. While the field is homogeneous on the axis of symmetry marked by the dashed line, distortions arise near the electrodes due to the constant electrical potential on a drift ring. As the ion trajectories will follow the streamlines of the electrical field due to the constant collisions with the drift gas, ions far from the axis of symmetry will take a longer, curved path (blue line) compared to the 
trajectories close to the axis (red line). Thus, the drift time of an ion depends not only on its mobility, but also on its radial position inside the drift tube, which can lead to ions with the same mobility arriving at different drift times [6]. As this phenomenon only causes delay, it leads to peak tailing and may reduce the analytical power of the drift tube due to broader and therefore lower peaks. A common method to ensure a clear Gaussian peak shape in the presence of field distortions is masking ions traveling far from the axis of symmetry. Unfortunately, this will lead to a lower signal amplitude and therefore a lower SNR.

\section{Numerical Field Simulations}

To generalize the simulations, the radius $r$ measured from the axis of symmetry as shown in Figure 1 will be described in terms of the normalized radius $\Gamma$, where $\Gamma$ is given by the radius $r$ divided by the inner tube radius $r_{i n}$.

$$
\Gamma=\frac{r}{r_{i n}}
$$

To begin with, it is necessary to determine the relationship between ring dimensions and peak shape. To do so, the ion distribution on the detector plane was calculated by simulating the ion trajectories in a given field distribution employing COMSOL Multiphysics using the Transport of Diluted Species module. The generated data can be used to evaluate the time related ion flux at each point of the detector in terms of the normalized radius $\Gamma$.

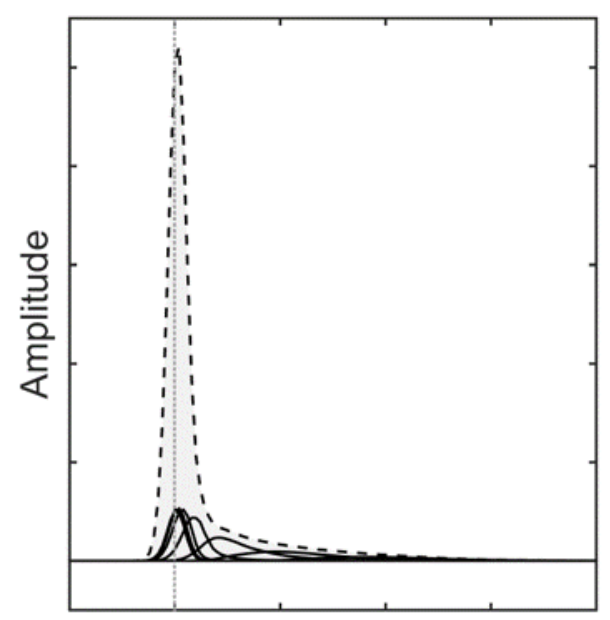

Drift time

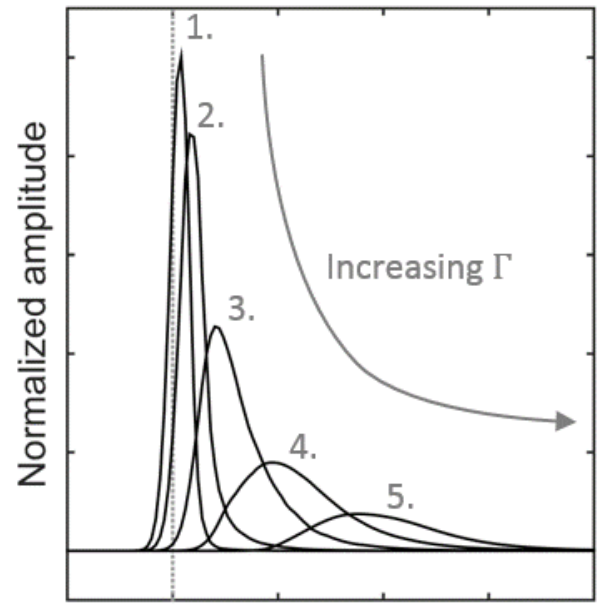

Drift time

Figure 2: On the left half, a simulation of the signal (dashed) resulting from distorted and delayed ion plugs (solid lines) reaching the detector is shown. On the right half, an enlargement of the peaks corresponding to the five most distorted ion plugs is shown. The data are generated by solving the ion motion in the field distribution presented in Figure 1.

Simulating the ion trajectories in an electrical field as the one shown in Figure 1 a peak shape as illustrated by the dashed line on the left half of Figure 2 can be obtained. Dividing this integrated signal into a number of discrete ion plugs (solid lines) moving on different normalized radiuses $\Gamma$ allows a detailed analysis of the relationship between the distribution of the electrically field and the overall peak shape. 
As illustrated in the right half of Figure 2, ion plugs closer to the axis of symmetry (1.) are nearly Gaussian shaped, while plugs moving close to the electrodes are delayed and asymmetrically broadened (5.). As most ion plugs move in a homogenous field, the integrated signal shown in the left half of Figure 2 is similar to a Gaussian peak with the expected drift time (dotted line), but reveals a small tailing. Thus, we define an effective radius $\Gamma_{\text {eff }}$ including all trajectories without significant negative impact on the peak shape. $\Gamma_{\text {eff }}$ therefore describes the effective usable radius of the drift tube.

To decide which of the illustrated ion plugs can be included without deforming the integrated peak, a mathematical measure for peak deformation is required. The resolving power $R_{p}$ might be the obvious candidate, as it is the most common criterion for the analytical performance of IMS and is also easy to measure. However, as it is based on the full width at half maximum, it will be insensitive to tailing which occurs far below the half height of the peak, such as the distortion shown on the left hand side of Figure 2. To circumvent this problem, we propose the ideality factor $\eta$ as the peak height $h_{\text {peak }}$ divided by the peak area $A_{\text {peak }}$ as given by Eq. ( 4 ).

$$
\eta=\frac{h_{\text {peak }}}{A_{\text {peak }}}
$$

As every kind of peak broadening, no matter its shape, must lead to a reduction of peak height at a given peak area, the ideality factor $\eta$ can be expected to react much more sensitively to the peak tailing shown in Figure 2. Normalization yields an ideality factor which is 1 for the Gaussian shaped peaks moving on the axis of symmetry and $<1$ for deformed peaks. For example, on the right hand side of Figure 2, the normalized values of $\eta$ would be 0.97 for peak 1 and 0.18 for peak 5 . The respective normalized resolving power would be 0.95 and 0.20 . However, when analyzing the resulting peak shown on the left hand side of Figure 2, the normalized ideality factor is 0.71 , while the normalized resolving power remains at 0.95. Thus, only the ideality provides a useful mathematical measure for peak deformation in this case.

Unfortunately, calculating either $\eta$ or $R_{p}$ requires numerical simulations of ion trajectories, which are much more time consuming than the calculation of the electrical field, as they must be solved for every time step of the simulation instead of only once. Thus, a parameter sweep sufficiently resolved for drift tube development would consume too much time to be feasible. Therefore, we propose a third method, which provides an approximation based on only the electrical field distribution $E_{(x, r)}$, which can be simulated within seconds or minutes. It is based on a two-step approach:

First, the peak position is calculated as a function of $\Gamma$. To do so, the inverse of the axial component of the electrical field multiplied by the inverse mobility $K$ is integrated along the length of the drift tube at the given $\Gamma$, which serves as a measure for the drift time along this line.

$$
\Delta t_{d_{(\Gamma)}}=\int_{0}^{L_{d}} \frac{1}{E_{(x, \Gamma)} \cdot K} d x
$$

This value is then normalized to the result at $\Gamma=0$, which is just the length of the drift tube $L_{d}$ squared divided by the drift voltage $U_{d}$ due to the homogenous field at this position. 


$$
t_{d}=\frac{L_{d}{ }^{2}}{U_{d} \cdot K}
$$

The result is a measure for the delay of the peak at the given $\Gamma$ compared to its ideal drift time $t_{d}$.

$$
\frac{\Delta t_{d_{(\Gamma)}}}{t_{d}}=\int_{0}^{L_{d}} \frac{U_{d}}{E_{(x, \Gamma)} \cdot L_{d}^{2}} d x
$$

Secondly, the radial component of the electrical field is evaluated as a measure for the interaction of peaks at different $\Gamma$. To do so, the radial electrical field distribution is integrated similarly to the method presented in eq. ( 7 ). The resulting voltage can be used to estimate the radial ion motion leading to interferences between ion plugs moving next to each other. Furthermore, by taking into account the radial diffusion, the estimation of the peak overlap can be improved even further. By using the drift times calculated with eq. ( 7 ) and assuming ideal Gaussian peaks only broadened by the additional diffusion due to the extra drift time, the resulting peak shape only gives a rough approximation of the reality (Figure 3, top). By considering the field driven and diffusive radial ion motion, the ion plugs become additionally broadened and slightly shifted (Figure 3, middle), which allows a much better approximation of the ion distribution obtained from an ion motion simulation (Figure 3 , bottom). The integrated signals in Figure 3 (middle, bottom) are calculated using all evaluated ion plugs, not only the four shown in the picture for clarity.

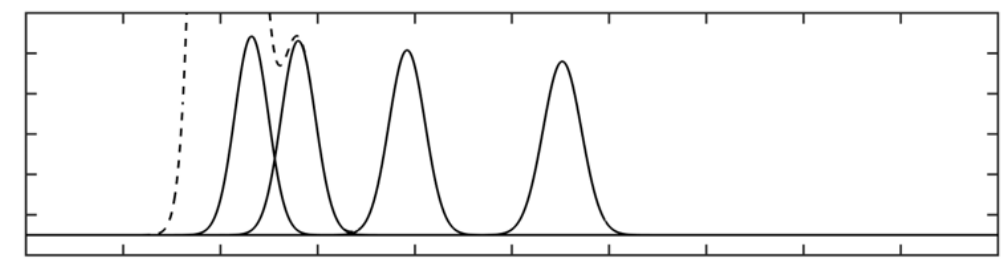

Axial field distortion only

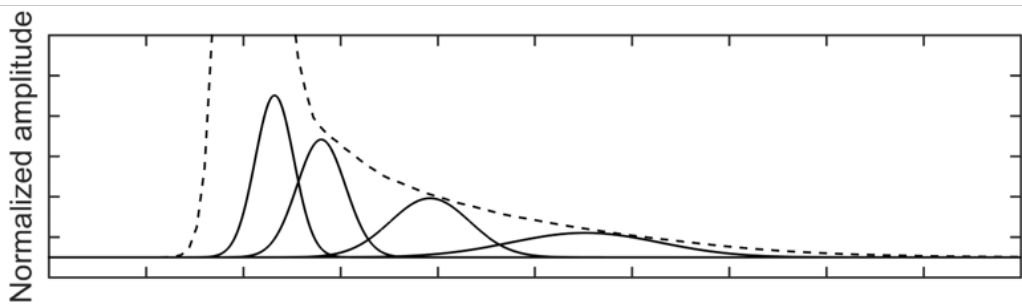

Including radial field distortion

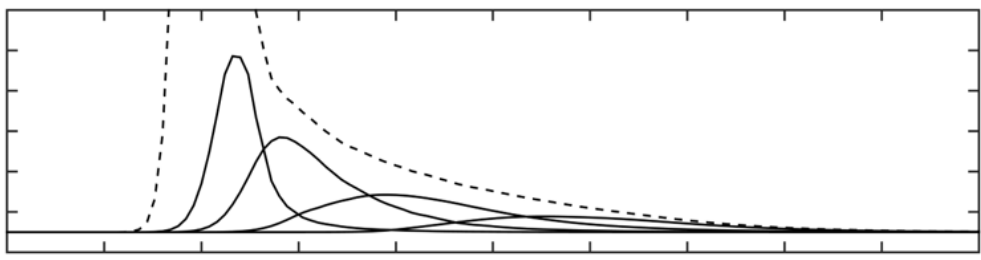

Normalized drift time

Figure 3: In the first panel, the ion distribution calculated by using only the axial distribution of the electrical field is shown. In the middle, the correction for the radial ion motion is included. The last panel shows the accurately computed ion distribution for comparison. 
These three measures for peak deformation are compared in Figure 4. As expected, the resolving power is too optimistic and even predicts no increasing deformation for very high $\Gamma$. The approximation from the electrical field only is on the other hand too pessimistic, but follows the ideality factor better in the regions of increasing deformation. Due to its vastly lower computational effort, it will be used for further analysis. The effectively usable radius $\Gamma_{\text {eff }}$ is defined as the value of $\Gamma$ with the strongest curvature, as it marks the bend in all three curves. The curvature is the highest $2^{\text {nd }}$ derivative, which is determined using a $3^{\text {rd }}$ order Savitzky-Golay-Filter.

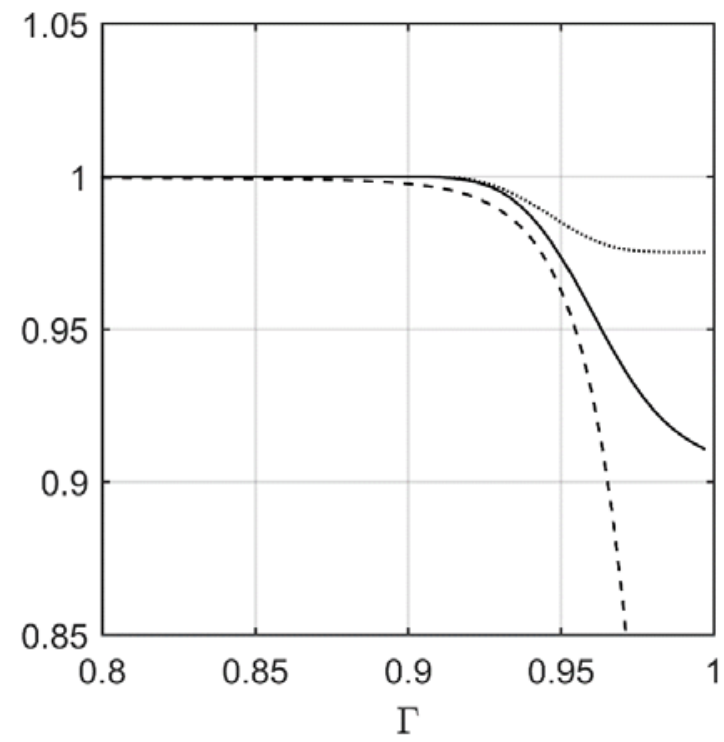

Figure 4: Normalized resolving power (dotted), ideality factor (solid) and electrical field approximation (dashed) as a function of the normalized radius $\Gamma$.

\section{Optimized Drift Ring Geometries}

It is now possible to compute the electrical field distribution for a number of different geometries with a 2D Field solver and evaluate the results by calculating the effective radius $\Gamma_{\text {eff. }}$ The simulations were performed using radially symmetric model similar to the one described in [7].

As the first step, the ring width $w_{\text {ring }}$ was swept. The ring width is given as the ratio between $w_{\text {ring }}$ and the pitch between two rings $p_{\text {ring }}$. Changing the absolute values at a constant ratio would not change the results, as it means just scaling up the design. Therefore, the ratios are the parameters of interest. As mentioned above, the complexity and therefore the cost of an IMS depends on the number of discrete elements. Thus, by maintaining a constant pitch $p_{\text {ring }}$ we ensure that the number of rings $n_{\text {ring }}$ is constant. 


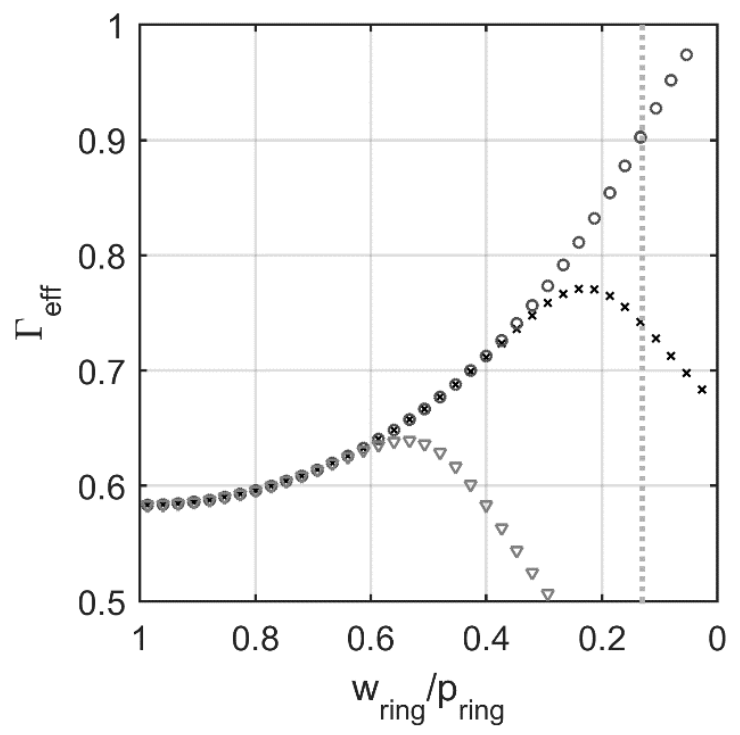

Figure 5: Effectively usable radius $\Gamma_{\text {eff }}$ as a function of $w_{\text {ring }} / p_{\text {ring }}$ for $d_{\text {ring }} / p_{\text {ring }}=2$ (circle), $d_{\text {ring }} / p_{\text {ring }}=1$ (cross) and $d_{\text {ring }} / p_{\text {ring }}=0.5$ (triangle). The inner tube radius is equal to the pitch $\left(r_{\text {in }} / p_{\text {ring }}=1\right)$.

As illustrated in Figure 5, decreasing the ring width $w_{\text {ring }}$ in relation to $p_{\text {ring }}$ will increase the effective useable radius $\Gamma_{\text {eff }}$ up to a certain point. This is expected since a conductive object has a constant electrical potential on its surface and therefor a large $w_{\text {ring }} / p_{\text {ring }}$ leads to a large potential difference between two rings. Accordingly, the distortion in the electrical field increases with increasing $w_{\text {ring }} / p_{\text {ring }}$. Therefore, best results can be achieved by using flat disks as ring geometries which correlates to the results presented by [8]. The main drawback is the missing shielding as shown in Figure 6 due to the poor ratio of ring depth $d_{\text {ring }}$ and the gap between two rings. Not only the shape of the drift field is distorted, but also its strength at this position is influenced by the grounded electrodes surrounding the dirft tube.

a)

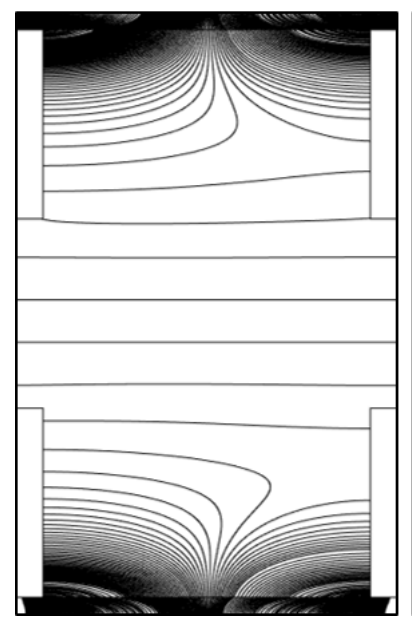

b)

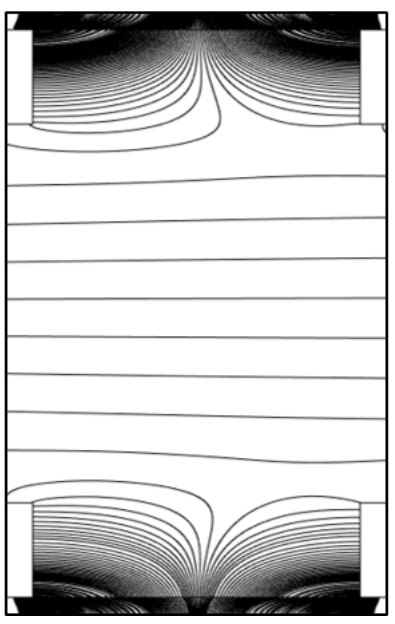

c)

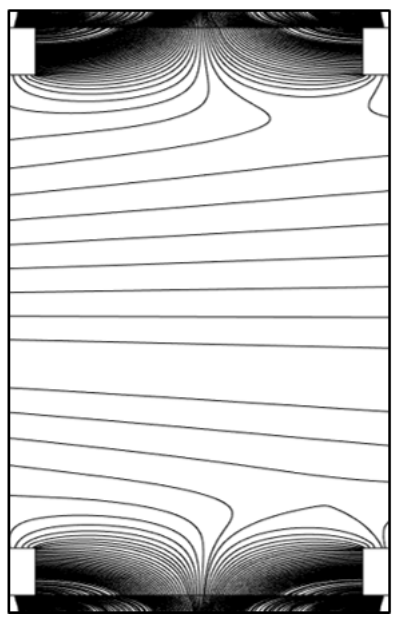

d)

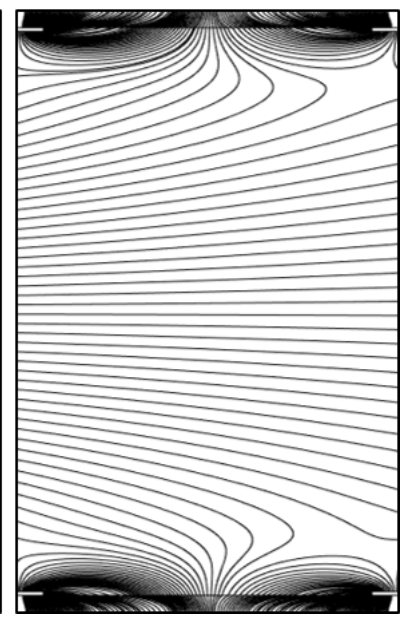

Figure 6: Simulation of the electrical field corresponding to $d_{\text {ring }} / p_{\text {ring }}$ equal $2,1,0.5$ and 0.13 (left to right) in presence of a grounded electrode surrounding the drift tube. 
This will lead to a decrease of the effectively usable radius $\Gamma_{\text {eff }}$ observed in Figure 5 above a certain $w_{\text {ring }} / p_{\text {ring, }}$ which depends on the $\mathrm{d}_{\text {ring }} / p_{\text {ring }}$. Thus, this effect could be overcome by raising the ring depth $d_{\text {ring }}$ with decreasing $w_{\text {ring. }}$. Nevertheless, in order to achieve an effectively usable radius $\Gamma_{\text {eff }}$ of more than 0.9 , a ring depth equal to twice the tube radius $r_{\text {in }}$ (Figure 5 , circles) would be needed. Therefore, at a constant number of drift rings building a compact IMS is not feasible, as wide rings require a large inner radius and thin rings require either also a large inner radius or a large ring depth. However, increasing the ring depth is only necessary to improve the shielding, which can also be achieved by decreasing the ring pitch $p_{\text {ring }}$ at the cost of increasing the number of rings $n_{\text {ring }}$ as shown in Figure 7. At a constant ring width $w_{\text {ring }}$ and depth $d_{\text {ring, }}$, the pitch $p_{\text {ring }}$ was swept.

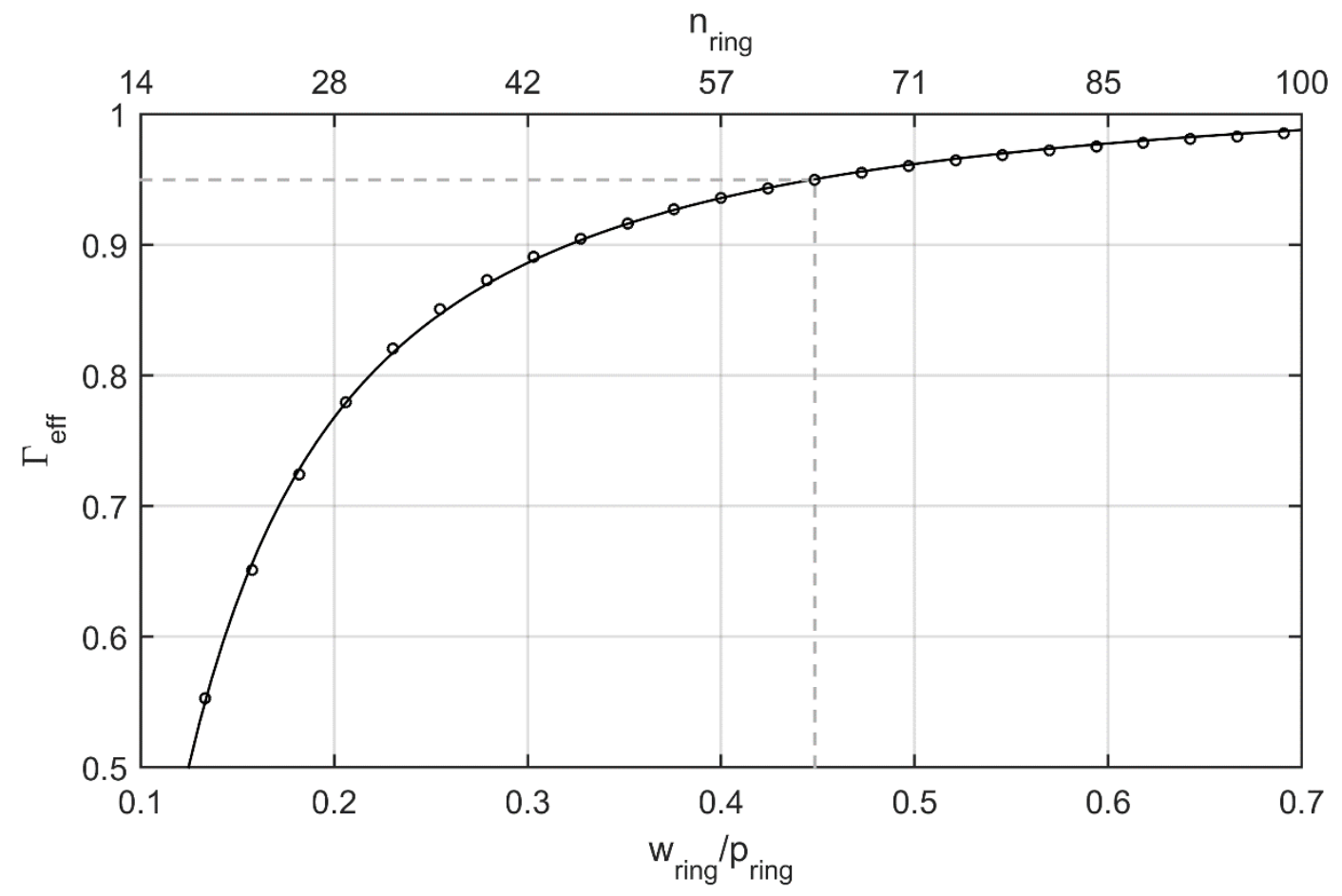

Figure 7: Effectively usable tube radius $\Gamma_{\text {eff }}$ (circles) as a function of $w_{\text {ring }} / p_{\text {ring }}\left(r_{\text {in }} / w_{\text {ring }}=7.5, d_{\text {ring }} / w_{\text {ring }}=0.1\right)$ and fit with a $x^{(3 / 2)}$ function (line). The second $x$-axis shows the number of rings $n_{\text {ring }}$ required in an IMS with a drift length of $100 \mathrm{~mm}$.

The large ratio of inner radius $r_{i n}$ to ring width $w_{\text {ring }}$ used for the simulation presented in Figure 7 equates $w_{\text {ring }} / p_{\text {ring }}=0.13$ as illustrated by the dotted line in Figure 5 . According to Figure 5 the used ratio should provide an extremely homogenous electrical field as long as the drift field distortions induced by the surrounding grounded electrodes can be completely shielded by the drift rings, e.g. with a large ring thickness as illustrated by Figure 5 (circles). However, decreasing the ring thickness will increase the influence of the present ground electrode which will entail a dramatic reduction of the effectively usable radius $\Gamma_{\text {eff }}$ as illustrated in Figure $6 \mathrm{~d}$ ). By reducing the pitch $p_{\text {ring, }}$, the effectively usable radius $\Gamma_{\text {eff }}$ can be increased at a constant outer radius even when a grounded electrode is in existence. Thus, if only a certain $\Gamma_{\text {eff }}$ is required regardless the number of rings, the outer radius can be decreased. However, now a suitable technique for accurately producing an extremely high number of closely spaced thin drift rings is required. 


\section{Printed Circuit Board IMS}

Well-known approaches for manufacturing drift tubes with high numbers of drift rings are the use of low temperature cofired ceramics (LTCC) [9] or resistive glass tubes (RGT) [10]. Simplified, drift tubes manufactured from LTCCs integrate drift rings and resistors into the housing by coating structured raw ceramics with conductive materials before baking the structure. While the LTCC still relies on discrete rings, the RGT is based on an isolating glass tube completely coated with a resistive material acting as both drift ring and resistor simultaneously. This is the equivalent of an infinite number of infinitely small drift rings. However, both approaches are expensive and inflexible due to the complex fabrication process. Another process, which is also able to produce finely spaced structures, but is already widespread and therefore cheap, is the manufacturing of printed circuit boards (PCB), which are part of virtually every electronic device today. The copper on their surface can be structured using lithographic processes to generate the necessary geometries for electrodes and feedthroughs with extremely high precision. Even though a printed circuit board IMS has been reported [11], this approach has not yet gained wide recognition. One of the main disadvantages of PCB manufacturing is the limitation to flat structures. Due to this, the setup reported in [11] uses only electrodes on the top and the bottom of the drift tube, causing deformations of the electrical field close to the sides.

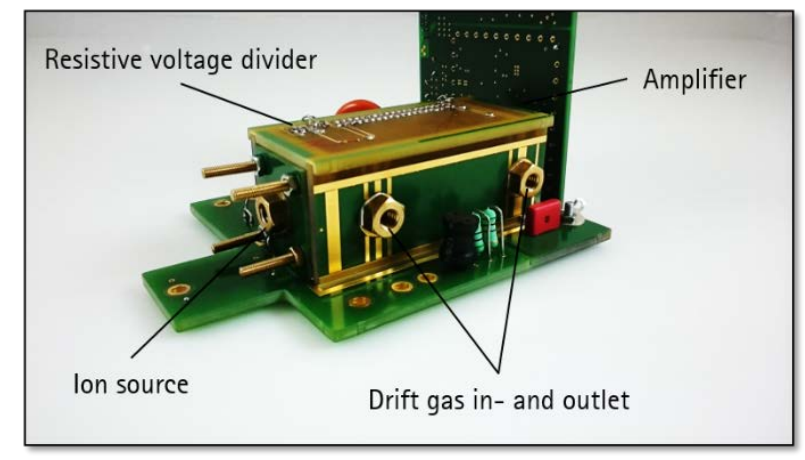

Figure 8: PCB-IMS with the resistive Voltage divider on top, ion source on the left and detector with attached amplifier on the right side.

To overcome this limitation, we developed a drift tube based on the results presented in the simulation section, which is manufactured completely from printed circuit boards. As it incorporates 45 electrodes on each of the four sides of the $50 \mathrm{~mm}$ long drift region, it can generate an extremely homogenous drift field while achieving a small size. The used PCBs are made of a glass-reinforced epoxy laminate (FR4) which is laminated with a $35 \mu \mathrm{m}$ film of copper. The basic layout of the PCB-IMS is similar to a typical stacked ring design, consisting of ionization region, drift region and a detector shielded by an aperture grid. A tritium source is used for ionization and ions are injected into the drift tube using a field switching (FS) shutter [12]. To minimize interferences on the ion signal, the current amplifier is directly attached to the backside of the PCB detector. The epoxy laminate acts as housing, isolator and as substrate for the drift rings simultaneously. The drift rings are connected to a resistive voltage divider placed on the outer layer of the drift tube by vertical interconnections between the inner and outer layers. Purified air is used as both drift gas and sample carrier. The experimental parameters of the setup are summarized in Table 1. 
Table 1: Experimental parameters

\begin{tabular}{|l|r|l|}
\hline \multicolumn{1}{|c|}{ Parameter } & \multicolumn{2}{|c|}{ Value } \\
\hline Drift length & 50 & $\mathrm{~mm}$ \\
\hline Source diameter & 10 & $\mathrm{~mm}$ \\
\hline Source activity & 300 & $\mathrm{MBq}$ \\
\hline Drift voltage & 3150 & $\mathrm{~V}$ \\
\hline Injection voltage & 500 & $\mathrm{~V}$ \\
\hline Repetition rate & 44 & $\mathrm{~Hz}$ \\
\hline Drift gas flow & 500 & $\mathrm{mls} \cdot \mathrm{min}^{-1}$ \\
\hline Sample gas flow & 20 & $\mathrm{mls}^{-1} \mathrm{~min}^{-1}$ \\
\hline \multirow{2}{*}{ Dew point of drift and sample gas } & -95.5 & ${ }^{\circ} \mathrm{C}$ \\
\cline { 2 - 3 } & 79.4 & $\mathrm{ppb}$ \\
\hline Operating pressure & 1018 & $\mathrm{mbar}$ \\
\hline Operating temperature & 21 & ${ }^{\circ} \mathrm{C}$ \\
\hline
\end{tabular}

\section{Results and Discussion}

In order to analyze the performance of the presented ion mobility spectrometer and to identify the optimal operating point, we measured the resolving power and signal-to-noise-ratio of the positive reactant ion peak as a function of the applied drift voltage. As shown in Figure 9, the resolving power increases with increasing drift voltage up to a maximum at a drift voltage of about $2.9 \mathrm{kV}$. The signal-tonoise-ratio, normalized to its value at $2.9 \mathrm{kV}$, increases continuously with increasing drift voltages. Its behavior agrees excellently with the analytical function calculated by [13], which is also shown in Figure 9. Therefore, the operating point is set to $3.2 \mathrm{kV}$ since the loss in resolving power is negligible compared to the increase of the signal-to-noise-ratio.

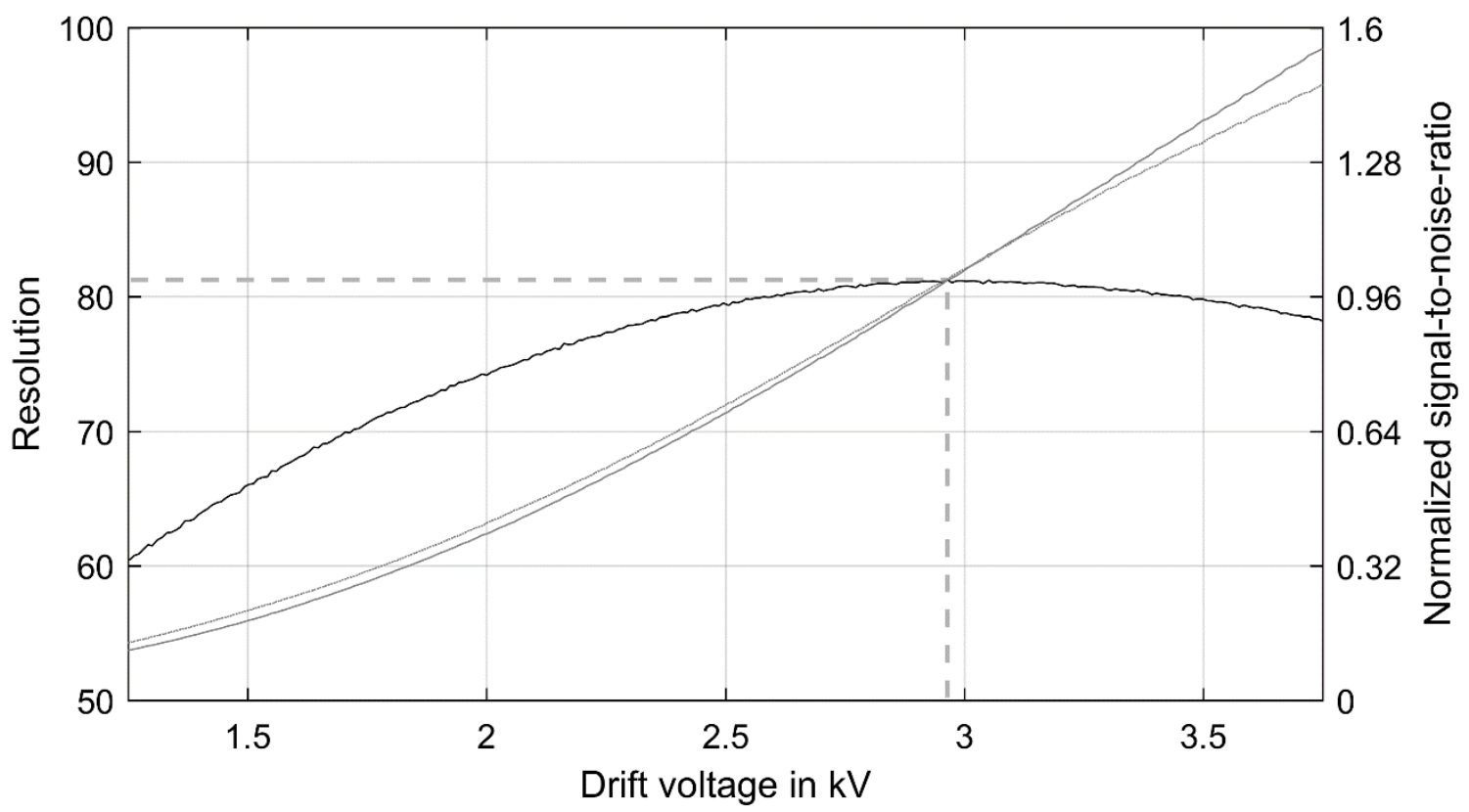


Figure 9: Resolving power (black), normalized measured (gray) and calculated SNR (gray, dotted) with the maximum resolving power of about 82 at a drift voltage of $2.96 \mathrm{kV}$ (dashed lines).

The resulting spectra in both positive and negative mode at a drift voltage of $3.2 \mathrm{kV}$ with 1024 averages are shown in Figure 10. The resolving power of the positive reactant ion peak $\left(\mathrm{RIP}^{+}\right)$is $R_{p}=82$, while the resolving power of the negative reactant ion peak (RIP) is $R_{p}=76$. As predicted by the field simulations and the approximation based on it, both peaks are nearly Gaussian shaped without tailing. The lower resolving power of the negative reactant ion peak can be attributed to a second reactant ion species, which is already visible in front of the main peak and can be separated at higher resolving powers [14].
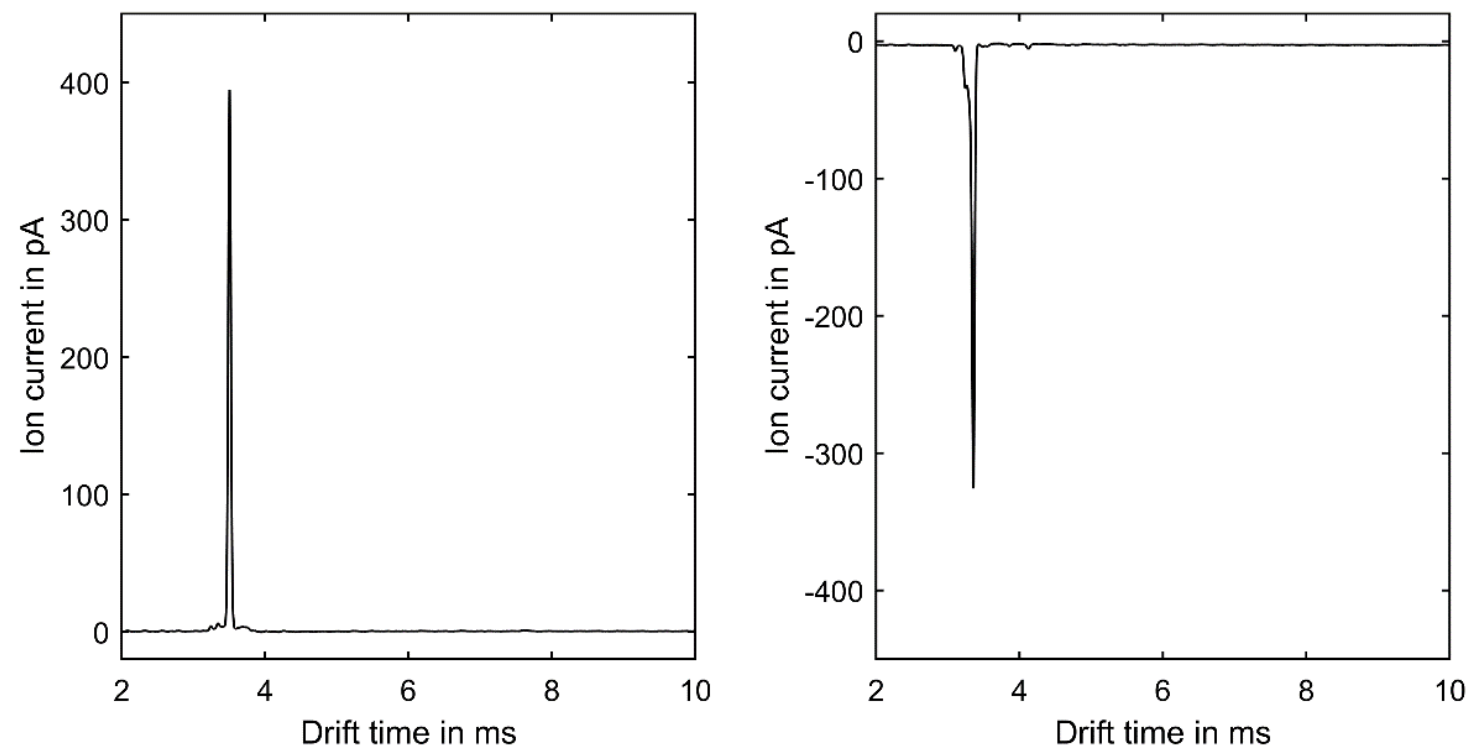

Figure 10: Spectra measured with the PCB-IMS. The positive $\mathrm{RIP}^{+}$(left) has a resolving power of $R_{p}=82$, the negative $\mathrm{RIP}^{-}$(right) a resolving power of $R_{p}=76$.

Figure 11 shows a measurement of $2 \mathrm{ppb}_{\mathrm{v}}$ acetone. The acetone dimer is clearly visible at a normalized drift time of 1.13, while the acetone monomer is only visible as a shoulder of the positive reactant ion peak due to the extremely dry conditions. Again, at higher resolving power, it would become visible [5]. The resulting limit of detection for a measurement time of one second using the acetone dimer is 80 $\mathrm{ppt}_{\mathrm{v}}$. The limit of detection for dimethyl methylphosphonate (DMMP) is $35 \mathrm{ppt}_{\mathrm{v}}$ for the monomer. 


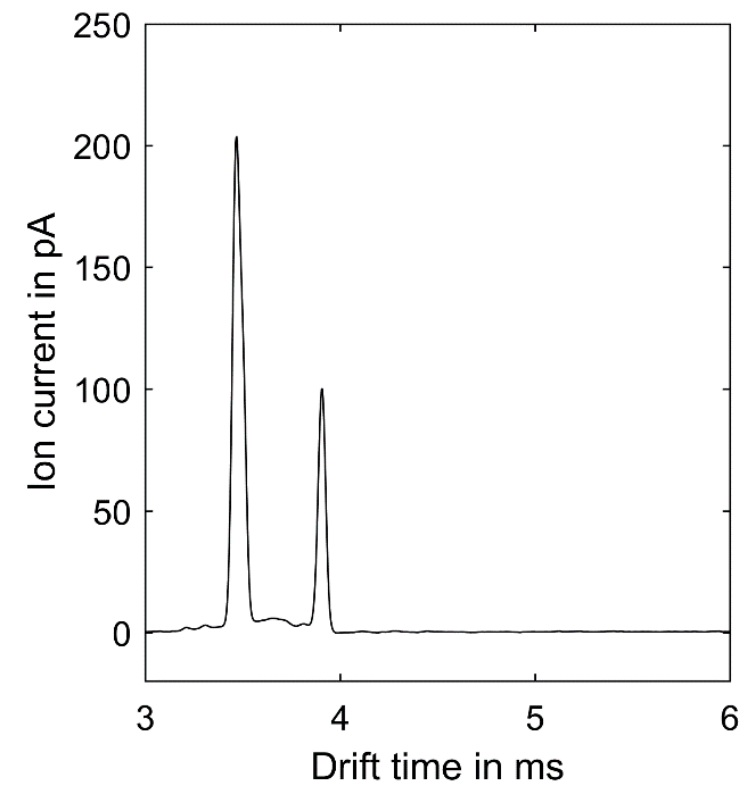

Figure 11: $2 \mathrm{ppb}_{\mathrm{v}}$ acetone 1024 averaged measured with the PCB-IMS.

Figure 12 shows a measurement of $4.3 \mathrm{ppb}_{\mathrm{v}}$ methyl salicylate in the negative mode. Only one product ion peak is formed at a normalized drift time of 1.39. The limit of detection for a measurement time of one second is $180 \mathrm{ppt}_{\mathrm{v}}$.

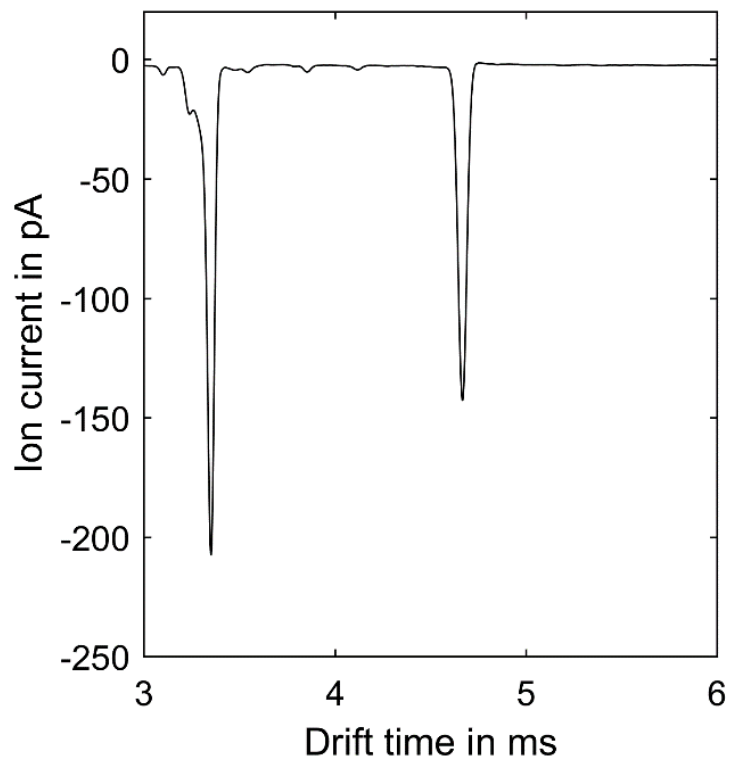

Figure 12: $4.3 \mathrm{ppb}_{\mathrm{v}}$ methyl salicylate measured with the PCB-IMS. 


\section{Conclusion}

In this work, we presented a method to estimate the influence of the electrical field distribution on the peak shape in a drift tube ion mobility spectrometer using only an electrical field simulation, but no ion motion simulation. All results are given as ratios between the parameters of interest. Therefore, this technique is applicable to any other drift tube design, providing a simple tool for estimating the correct dimensions of a drift cell. Using the developed models, we designed an ion mobility spectrometer based on printed circuit boards only. This device offers a resolving power of about 80 and simultaneously limits

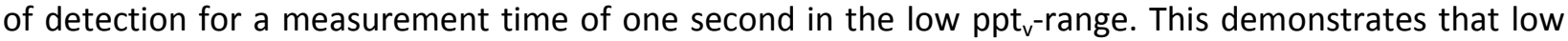
cost, compact, high performance drift tubes are indeed feasible. These could offer a basis for the design of overall low cost ion mobility spectrometer systems with high analytical power, bringing ion mobility spectrometry into more applications.

\section{References}

1. Eiceman GA, Karpas Z, Hill HH (2013) Ion mobility spectrometry, 3rd edn. CRC Press, Boca Raton

2. Fink T, Baumbach JI, Kreuer S (2014) Journal of breath research 8:1-11

3. O’Donnell RM, Sun X, Harrington, Peter de B. (2008) TrAC Trends in Analytical Chemistry 27:44-53

4. Borsdorf H, Eiceman GA (2006) Applied Spectroscopy Reviews 41:323-375

5. Kirk AT, Allers M, Cochems P, Langejuergen J, Zimmermann S (2013) The Analyst 138:5200-5207

6. Rokushika S, Hatano H, Baim MA, Hill HH (1985) 57:1902-1907

7. Langejuergen J, Cochems P, Zimmermann S (2012) 15:247-255

8. Soppart O, Baumbach JI (2000) Measurement Science and Technology 11:1473-1479

9. Pfeifer KB, Rumpf AN (2005) 77:5215-5220

10. Kaplan K, Graf S, Tanner C, Gonin M, Fuhrer K, Knochenmuss R, Dwivedi P, Hill HH (2010) Analytical chemistry 82:9336-9343

11. Eiceman GA, Schmidt H, Rodriguez JE, White CR, Krylov EV, Stone JA (2007) Instrumentation Science \& Technology 35:365-383

12. Kirk AT, Zimmermann S (2014) International Journal for lon Mobility Spectrometry 17:131-137

13. Kirk AT, Zimmermann S (2015) International Journal for Ion Mobility Spectrometry 18:129-135

14. Kirk AT, Zimmermann S (2015) International Journal for Ion Mobility Spectrometry 18:17-22 